\section{APL: ATRA und Arsentrioxid in Kombination}

Zum Vergleich von All-trans-Retinsäure (ATRA) plus Arsentrioxid (ATO) mit ATRA/Chemotherapie (CHT) in der Erstlinie bei akuter Promyelozyten-Leukämie $(\mathrm{APL})$ mit niedrigem und intermediärem Risiko liegen finale Ergebnisse vor.

$\mathrm{M}$ it dem bisherigen Standard ATRA/ CHT lassen sich zwar hohe Heilungsraten erzielen, doch der Preis sind oft schwere hämatologische Nebenwirkungen und - bei $2 \%$ der Patienten Zweitmalignome. ATO ist derzeit als Mo-

notherapie für die rezidivierte oder refraktäre APL zugelassen. In der randomisierten Phase-III-Studie APL0406 hatte sich bei den ersten 156 Patienten angedeutet, dass ATRA/ATO der Standardkombination ATRA/CHT bei neu

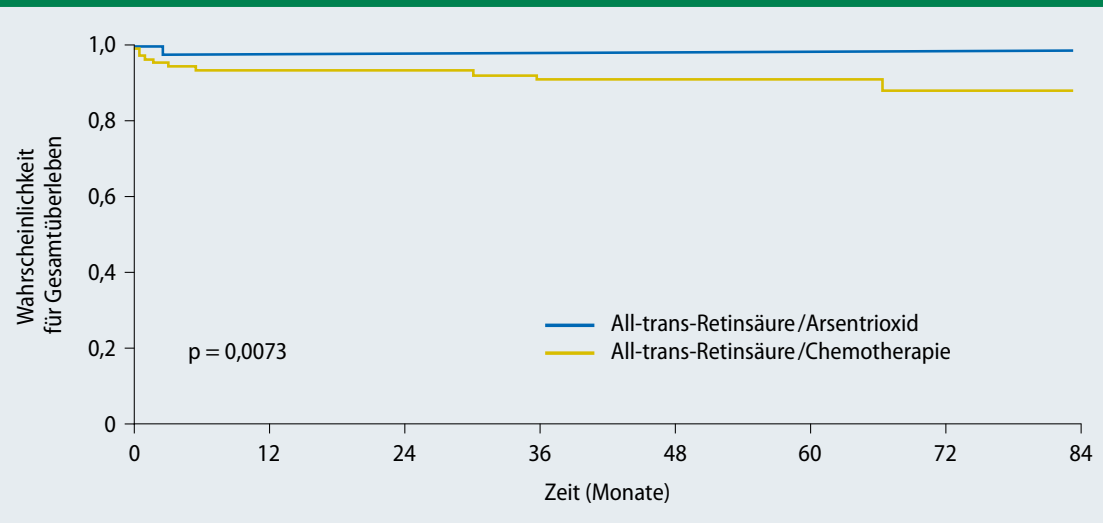

Abb. 1: ATRA/ATO führte zu einer höheren Überlebenswahrscheinlichkeit.

\section{CML: Zweite Generation der TKI wirksamer}

In der DASISION-Studie hatte sich Dasatinib in der Ersttherapie der chronischen myeloischen Leukämie (CML) gegenüber Imatinib als überlegen erwiesen. Nun wurden die finalen Ergebnisse nach 5 Jahren veröffentlicht.

n der randomisierten Phase-III-Studie
DASISION hatten 519 Patienten mit
neu diagnostizierter CML in der chroni-
schen Phase $(\mathrm{CP})$ entweder den Zweitge-
nerations-Tyrosinkinasehemmer (TKI)
Dasatinib $(100 \mathrm{mg} / \mathrm{d}$ ) oder Imatinib
(400 mg/Tag) erhalten. Bei suboptima-
lem Ansprechen nach 3-18 Monaten war
eine Steigerung auf $140 \mathrm{mg} /$ Tag Dasa-
tinib oder $600-800 \mathrm{mg} / \mathrm{Tag}$ Imatinib
erlaubt. Primärer Endpunkt war das
komplette zytogenetische Ansprechen
(CCyR) nach 12 Monaten, sekundäre
Endpunkte die Gesamtzeit bis zur CCyR
und ihre Dauer, die Rate an guten mole-
kularen Remissionen (MMR), die Zeit

bis zur MMR, das progressionsfreie (PFS) und das Gesamtüberleben (OS). Nach 5 Jahren betrugen die kumulativen MMR- und MR ${ }^{4,5}$-Raten $76 \%$ und $42 \%$ unter Dasatinib sowie $64 \%$ und $33 \%$ unter Imatinib ( $p=0,0022$ und $p=0,0251)$. Die 5-Jahres-OS-Raten betrugen $91 \%$ (Dasatinib) und 90\% (Imatinib; Hazard Ratio [HR] 1,01). Mehr mit Imatinib behandelte Patienten starben an den Folgen der CML (17 vs. 9), das 5-Jahres-OS unterschied sich jedoch nicht signifikant (HR 0,53; p = 0,1192). Auch die Rate für das 5-Jahres-PFS war in beiden Gruppen ähnlich hoch (85 vs. $86 \%$; HR 1,06). Patienten, bei denen BCR-ABL1 nach 3 diagnostizierter APL ohne hohes Risiko nicht unterlegen ist oder sogar Vorteile hat. Nun liegen die finalen Ergebnisse vor.

In der Induktion erreichten alle $127 \mathrm{~Pa}-$ tienten im ATRA/ATO- und 132 von 136 im ATRA/CHT-Arm eine komplette Remission $(p=0,12)$. Von 234 Patienten waren nach 3 Konsolidierungszyklen alle im ATRA/ATO-Arm MRD-negativ, während $2(1,7 \%) \mathrm{im}$ ATRA/CHT-Arm positiv getestet wurden. Nach median 40,6 Monaten betrug die Rate für das ereignisfreie Überleben $97,3 \%$ im ATRA/ATOvs. $80 \%$ im ATRA/CHT-Arm (p $<0,001)$, die kumulative Rezidivrate $1,9 \%$ versus $13,0 \%(\mathrm{p}=0,0013)$ und die 50-MonatsÜberlebensrate $99,2 \%$ versus $92,6 \%$ $(\mathrm{p}=0,0073)($ Abb. 1).

Fazit: Die Daten zeigen, dass der Erstlinien-Einsatz von ATRA/ATO bei APL mit niedrigem bis intermediärem Risiko Vorteile bringen kann. Die Kombination hat offenbar einen länger dauernden antileukämischen Effekt. Kathrin von Kieseritzky

Platzbecker U et al. Improved Outcomes With Retinoic Acid and Arsenic Trioxide Compared With Retinoic Acid and Chemotherapy in NonHigh-Risk Acute Promyelocytic Leukemia: Final Results of the Randomized Italian-German APL0406 Trial. J Clin Oncol. 2016 Jul 11. [Epub ahead of print].

Monaten auf $\leq 10 \%$ gesunken war $(84 \%$ unter Dasatinib, 64\% unter Imatinib), erreichten in den 5 Jahren häufiger eine CCyR, MMR sowie MR ${ }^{4,5}$ und hatten ein besseres PFS und OS als die Patienten mit BCR-ABL1 > 10\% nach 3 Monaten. Zudem ging bei ihnen die Krankheit seltener in die akzelerierte/Blastenphase über (3\% vs. $14-15 \%)$.

In den 5 Jahren wurden keine unbekannten Nebenwirkungen registriert.

Fazit: Die finalen Ergebnisse stützen den Einsatz von Dasatinib als effiziente und sichere Erstlinientherapie der CML-CP, auch wenn sich beide im PFS und OS nicht unterschieden. Patienten sprachen unter Dasatinib molekular früher an als unter Imatinib.

Kathrin von Kieseritzky

Cortes J E et al. Final 5-Year Study Results of DASISION: The Dasatinib Versus Imatinib Study in Treatment-Naive Chronic Myeloid Leukemia Patients Trial. J Clin Oncol. 2016;34(20):2333-40. 